\title{
NOTAS SOBRE LA BRIOFLORA BALEAR, 5
}

\author{
J.A. ROSSELLO
}

\begin{abstract}
RESUMEN: Se citan algunos briófitos que son novedad para el catálogo florístico balear: Marchantia paleacea Bertol., Sphaerocarpos texanus Aust., Bryum pseudotriquetrum (Hedw.) Gaertn et al., Orthotrichu affine Brid., Pseudoleskeella catenulata (Schrad.) Kindb. y Sematophyllum substrumulosum (Hampe) Britt. Se mencionan especies que no habian sido indicadas en la flora de Ibiza (9), Formentera (1) y Mallorca (1).
\end{abstract}

SUMMARY: The following bryophytes are quoted for the first time in the flora of the Balearic Islands: Marchantia paleacea Bertol., Sphaerocarpos texanus Aust., Bryum pseudotriquetrum (Hedw.) Gaertn. et al., Orthotrichum affine Brid., Pseudoleskeella catenulata (Schrad.) Kindb. and Sematophyllum substrumulosum (Hampe) Britt. Some species are new for the flora of Ibiza (9), Formentera (1) and Mallorca (1).

Con esta nota continuamos la serie de aportaciones corológicas cuyo fin es la elaboración de un catálogo floristico balear (Rosselló, 1981; 1984; 1985a; 1985b). Presentamos los resultados más interesantes de las herborizaciones briológicas realizadas en 1984 y 1985 en Mallorca, Ibiza y Formentera. En Mallorca hemos continuado la exploración de la Sierra de Tramuntana. Dado el escaso conocimiento briológico que poseemos aun de Ibiza hemos recolectado abundante material, principalmente de la zona montañosa del suroeste y de algunas fuentes y torrentes de la parte norte.

La nomenclatura de hepáticas y musgos, que están ordenados alfabéticamente, sigue a Düel1 (1983) y a Corley et al. (1981) respectivamente, con la excepción de Zygodon rupestris, para el que seguimos la adoptada por Karttunen (1984).

Las localidades indicadas en el texto van acompañadas de su retículo UTM $(10 \mathrm{x} 10 \mathrm{~km})$ correspondiente. Las novedades corológicas para el archipiélago balear van señaladas con dos asteriscos ( $\left.*^{*}\right)$, y las que constituyen novedad insular con uno (*). Los pliegos testimonio de las especies enumeradas se encuentran en el herbario particular del autor y en el herbario de la Universidad de Palma de Mallorca. 
HEPATICAE

Cololejeunea calcarea (Libert.) Schiffn.

Mallorca: muy rara en el bosque de Son Massip ( $D E$ 80), en los tocones de Q. ilex, entre céspedes de Metzgeria furcata y Lejeunea cavifolia. Estéril. Conocida anteriormente de Tomir, Ternelles y Cala S. Viçenç (Koppe, 1965).

Frullania tamarisci (L.) Dum.

Mallorca: Na Burguesa (DD 68), Mola de Planicie (DD 59), Son Massip (DE 80) sobre tierra; alrededores de Pollensa en Sa Vall d'En March (DE 91) epífita sobre Olea europaea. Estéril. Se conocía de Sóller, Ternelles y Bunyola (Koppe, 1965).

Jungermannia atrovirens Dum.

Mallorca: se encuentra sumergida en las canales de Ternelles (EE 01), $\mathrm{pH}$ del agua 7,5. Estéril. Las localidades conocidas de esta especie eran las de Tossals y Massanella (Rosselló, 1981).

Marchantia paleacea Bertol. (**)

Mallorca: piedras húmedas de un torrente en las inmediaciones de Mortitx (DE 91), junto a Bryum pseudotriquetrum. Con propágulos.

Pellia endiviifolia (Dicks.) Dum. (*)

Ibiza: sobre rocas en la Font des Tur y en el Torrent des Buscastell (CD 52). Estéril.

Petalophyllum ralfsii (Wills.) Nees \& Gott. (*)

Ibiza: raro en los rellanos arcillosos de Cala d'Aubarca (CD 52). Estéril. Formentera: escaso en los torrentes de la Mola (CC 78). Estéril.

Phaeoceros laevis (L.) Prosk. (*)

Mallorca: sobre las arcillas de la Marina de Son Serra (ED 29), junto a Cladonia sp. pl. Fértil. Tradicionalmente se ha señalado la presencia de P. laevis en Menorca (Rodríguez, 1875; Bolós et al., 1970) en tanto que en Mallorca se ha indicado el P. bulbiculosus (Brot.) Prosk. (Casas, 1956; Koppe, 1965; Sloover, 1967). La revisión de los ejemplares recogidos por Sloover en La Calobra (BCB 235) corresponden a P. laevis (los ejemplares de Formentor, BCB 234, son estériles). Cabe señalar que la cita de Casas (1956) de P. bulbiculosus procede también de La Calobra, pero no hemos encontrado en $\mathrm{BCB}$ ningún testimonio de herbario. No nos ha sido posible hasta el momento examinar el herbario de F. Koppe (Duisburgo), pero será imprescendible para confirmar la presencia de P. bulbiculosus en Baleares.

Plagiochasma rupestre (Forst.) Steph. (*)

Ibiza: abundante en los rellanos en el Puig de s'Argentera (CD 71) y en la Cala d'Aubarca (CD 52).

Sphaerocarpos texanus Aust. (**)

Mallorca: en las arcillas del Clot d'Aubarca, en las inmediaciones de 
Lluc (DE 80) donde escasea. Fértil. Esta especie tiene en España una distribución reducida (fig. 1).

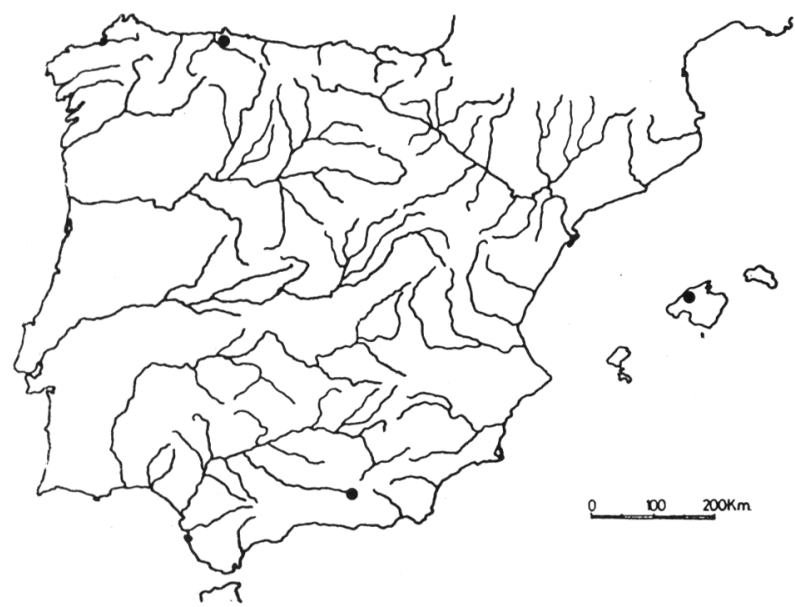

Figura 1. Distribución conocida en España de Sphaerocarpos texanus Aust.

\section{MUSCI}

Bryum pseudotriquetrum (Hedw.) Gaertn. et al. (**)

Mallorca: rocas húmedas en un torrente en las cercanías de Mortitx (DE 91), con Marchantia paleacea y Pellia endiviifolia. Estéril.

Ctenidium molluscum (Hedw.) Mitt. (*)

Ibiza: rara en Ses Roques Altes (CD 41), bajo arbustos de Juniperus phoenicea y Pistacia lentiscus. Estéril.

Funaria curviseta (Schwaegr.) Milde (*)

Ibiza: Puig de s'Argentera (CD 71), en los rellanos arcillosos, junto a Riccia sp. Con esporófitos.

Grimmia orbicularis Bruch ex Wills. (*)

Ibiza: sobre rocas en el lecho del Torrent de s'Aigo (CD 50). Fértil.

Isothecium striatulum (Spruce) B.S. \& G.

Mallorca: en las paredes verticales del Torrent de Coa Negra (DD 79). Fértil. Conocido del Puig Major (Nicholson, 1907), Mal Pas y Artá (Sloover, 1967) aunque los ejemplares de estas dos últimas localidades, que han sido revisados por C. Casas (in schedae, BCB 4296, 4300), corresponden a Scorpiurium circinatum. 
Leptodon smithii (Hedw.) Web. \& Mohr (*) Estéril.

Ibiza: en las grietas de las rocas en la Cala d'Aubarca (CD 52), raro.

Orthotrichum affine Brid. (**)

Mallorca: epifito sobre Q. ilex en la Serra d'Alfabia (DE 79), muy raro, a unos $900 \mathrm{~m}$. Con esporófitos.

Orthotrichum diaphanum Brid. (*)

Ibiza: en el tronco de O. europaea en las inmediaciones de la Cala d'Aubarca (CD 52).

Plagiomnium rostratum (Schrad.) T. Kop.

Mallorca: raro en la entrada de una cavidad vertical en el Puig Major (DE 80) a unos $1300 \mathrm{~m}$. Con esporófitos.

Pseudoleskeella catenulata (Schrad.) Kindb. (**)

Mallorca: en las paredes verticales calcáreas del Puig de Massanella (DE 80) a $1200 \mathrm{~m}$; sobre rocas y epifita de Acer granatense en el Puig Major (DE 80) entre 800 y $1300 \mathrm{~m}$. Estéril.

Sematophyllum substrumulosum (Hampe) Britt. (**)

Mallorca: sobre un tronco en descomposición, probablemente de $\mathrm{P}$. halepensis, en el lecho del Torrent de Coa Negra (DD 79), muy rara. Con esporófitos. Esta especie calcífuga, con distribución mediterráneo-atlántica, tiene una distribución reducida en España (fig. 2).

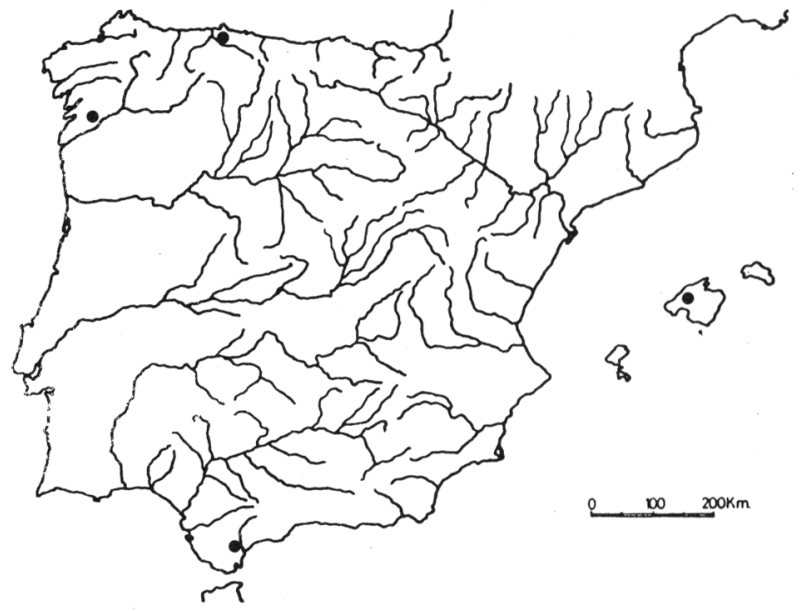

Figura 2. Distribución conocida en España de Sematophyllum substrumulosum (Hampe) Britt.

Zygodon rupestris Schimp ex

Ibiza: epifito sobre P. halepensis, J. phoenicea y P. lentiscus en Ses Roques Altes (CD 41). 


\section{AGRADECIMIENTOS}

Desearíamos agradecer a N. Torres y J. Rita la compañía y estímulo durante las herborizaciones por Ibiza y Mallorca, así como a C. Casas y M. Brugués por la identificación de algunas especies dudosas.

\section{BIBLIOGRAFIA}

BOLOS, O., MOLINIER, R. \& MONSERRAT, P. -1970- Observations phytosociologiques dans l'île de Minorque. Acta Geobot. Barcin. 5: $150 \mathrm{pp}$.

CASAS, C. -1956- Contribución al estudio de la flora briológica balear. Pharmacia Medit. 1: 1-16.

CORLEY, M.F.V., CRUNDWELL, A.C., DULL, R., HILL, M.O. \& SMITH, A.J.E. -1981- Mosses of Europe and the Azores; an annotated list of species, with synonyms from the recent literature. J. Bryol. 11: 609-689.

DUELL, R. -1983- Distribution of the European and Macaronessian liverworts (Hepaticophytina). Bryol. Beitr. 2: 1-115.

KARTTUNEN, K. -1984- Zygodon gracilis, Z. rupestris and Z. dentatus, comb. nova (Musci, Orthotrichaceae). History and nomenclature. Ann. Bot. Fennici 21: 343-348.

KOPPE, F. -1965- Bryologische Beobachtungen auf der Insel Mallorca. Bot. Not. 118: 25-48.

NICHOLSON, W.E. -1907- Contributions to a list of the mosses and hepatics of Majorca. Rev. Bryol. 34: 1-6.

RODRIGUEZ, J.J. -1875- Catálogo de los musgos de las Baleares. Anal. Soc. Esp. Hist. Nat. 4: 41-51.

ROSSELLO, J.A. -1981- Notes sobre la brioflora balear. 1. Briòfites noves per a Balears. Boll. Soc. Hist. Nat. Bal. 25: 39-52.

ROSSELLO, J.A. -1984- Notes sobre la brioflora balear. 2. Boll. Soc. Hist. Nat. Bal. 28: 135-137.

ROSSELLO, J.A. -1985a- Notes sobre la brioflora balear. 3. Collect. Bot. $16: 63-66$

ROSSELLO, J.A. -1985b- Notas sobre la brioflora balear.4. Acta Bot. Malacitana. 11: 72-82.

SLOOVER, J.L. -1967- Quelques bryophytes récueillies à Majorque. Les Nat. Belgues. 48: 389-394. 